\title{
CT features of small bowel obstruction caused by concurrent, ipsilateral obturator and inguinal hernix.
}

\section{Fontanella G. MD FRSA', Borrelli S. RTR², Russo M. MD',} Biondo F.G. MD ${ }^{1}$

1 Ospedale Sacro Cuore di Gesù FBF, Benevento, Italy

2 UPMC Hillman Cancer Center Villa Maria, Mirabella Eclano, Italy

Contact: giovanni.fontanella@hotmail.com

\section{KEYWORDS}

Hernia, radiology, bowel, obturator, CT, surgery, bowel obstruction, abdomen, pelvis.

\section{ABSTRACT}

Obturator herniæ are a quite rare type of abdominal hernia, accounting for less then $1 \%$ of all abdominal wall hernias, that arise when abdominal content, usually bowel loops and omentum, protrudes through the anterosuperior portion of the obturator foramen into the obturator canal, leading to small bowel obstruction in more than $90 \%$ of patients. Often signs and symptoms of obturator herniæ are quite unspecific; considering that, at the same time, patients with an obturator hernia are generally elderly, with concurrent conditions, this might cause delays in the diagnosis and treatment and produces the high postoperative mortality rates that obturator hernix yield

\section{INTRODUCTION}

Obturator herniæ are a quite rare type of abdominal hernia, accounting for less then $1 \%$ of all abdominal wall hernias and for $0.2-1.6 \%$ of patient with a small bowel obstruction [1]. Such condition arises when abdominal content, usually bowel loops and omentum, protrudes through the anterosuperior portion of the obturator foramen into the obturator canal, leading to small bowel obstruction in more than $90 \%$ of patients [1]. The obturator canal is the only portion of the obturator foramen that is not obliterated by the membrane of the same name and hosts the obturator neurovascular bundle; it is, for this reason, a locus minoris resistentiae, through which obturator hernias are formed, generally in elderly, multiparous women [2]. 
Inguinal herniæ are much more common, accounting for the vast majority, 80\%, of all abdominal wall hernix; considering the lifetime risk of developing an abdominal wall hernia is $5 \%$, the combination of obturator and inguinal herniæ in the same patient, as in the case we are presenting here, is extremely rare [3].

Despite the fact that Computed Tomography offers the possibility of a quick, correct preoperative diagnosis, often signs and symptoms of obturator herniæ are quite unspecific; considering that, at the same time, patients with an obturator hernia are generally elderly, with concurrent conditions, this might cause delays in the diagnosis and treatment and produces the high postoperative mortality rates that obturator herniæ yield $[2,4]$.

\section{IMAGING}

From a radiologic point of view, considering that CECT is a useful tool for a prompt diagnosis of the condition, which is often overlooked [2,5], it is of utmost importance to detect and report the anomaly, because of the serious implications that have already been described $[2,5-6]$.

In our pictorial essay, we will discuss as an example the case of a 92-year old woman with an acute abdomen and suspect of small bowel obstruction, referred to our Radiology Department for a CT scan. As seen in Figure 1, the presence of a small bowel obstruction was quickly detected, due to the presence of small bowel luminal dilation and multiple fluid-gas levels, confirmed by the fact that the colon appeared as collapsed, from cecum to rectum. The search for luminal caliber transition points highlighted the presence of both a proximal right obturator hernia (Fig.2) and a distal right inguinal hernia (Fig.3). Both herniæe were made up of small bowel loops and omentum, and were on the right side; obturator herniæ are usually found on the right side and more rarely on the left, because the presence of the sigmoid colon offers some kind of protection to the left obturator canal [7].

Figure 2 clearly shows were to look for obturator herniæ, considering the hernia arises protruding through the internal obturator muscle and obturator canal; obturator herniæ lie upon the pectineus muscle, beneath the external obturator muscle - this space needs to be carefully evaluated in the case of bowel obstruction in order to avoid diagnostic errors. 


\section{CONCLUSIONS}

Obturator herniæ are a quite rare condition and the concurrent presence of an inguinal hernia is an even rarer possibility. However, when present, obturator herniæ are at high risk of bowel loop obstruction, strangulation, post-surgical complication and even death, especially as patients are typically elderly individuals with pre-existing conditions $[2,8,9]$.

As obturator herniæ tend to be often clinically and radiologically overlooked, in the case of small bowel obstruction at CT, the space between the pectineus and the external obturator muscle has to be checked for the presence of bowel loops, in order to avoid missing a crucial diagnosis [9].

\section{REFERENCES}

[1]. Park, Jinyoung MD, PhD Obturator hernia, Medicine: August 21, 2020 - Volume 99 - Issue 34 - p e21701 doi: 10.1097/MD.0000000000021701 
[2]. Gilbert JD, Byard RW. Obturator hernia and the elderly. Forensic Sci Med Pathol. 2019 Sep;15(3):491-493.Mnari W, Hmida B, Maatouk M, et al. Strangulated obturator 


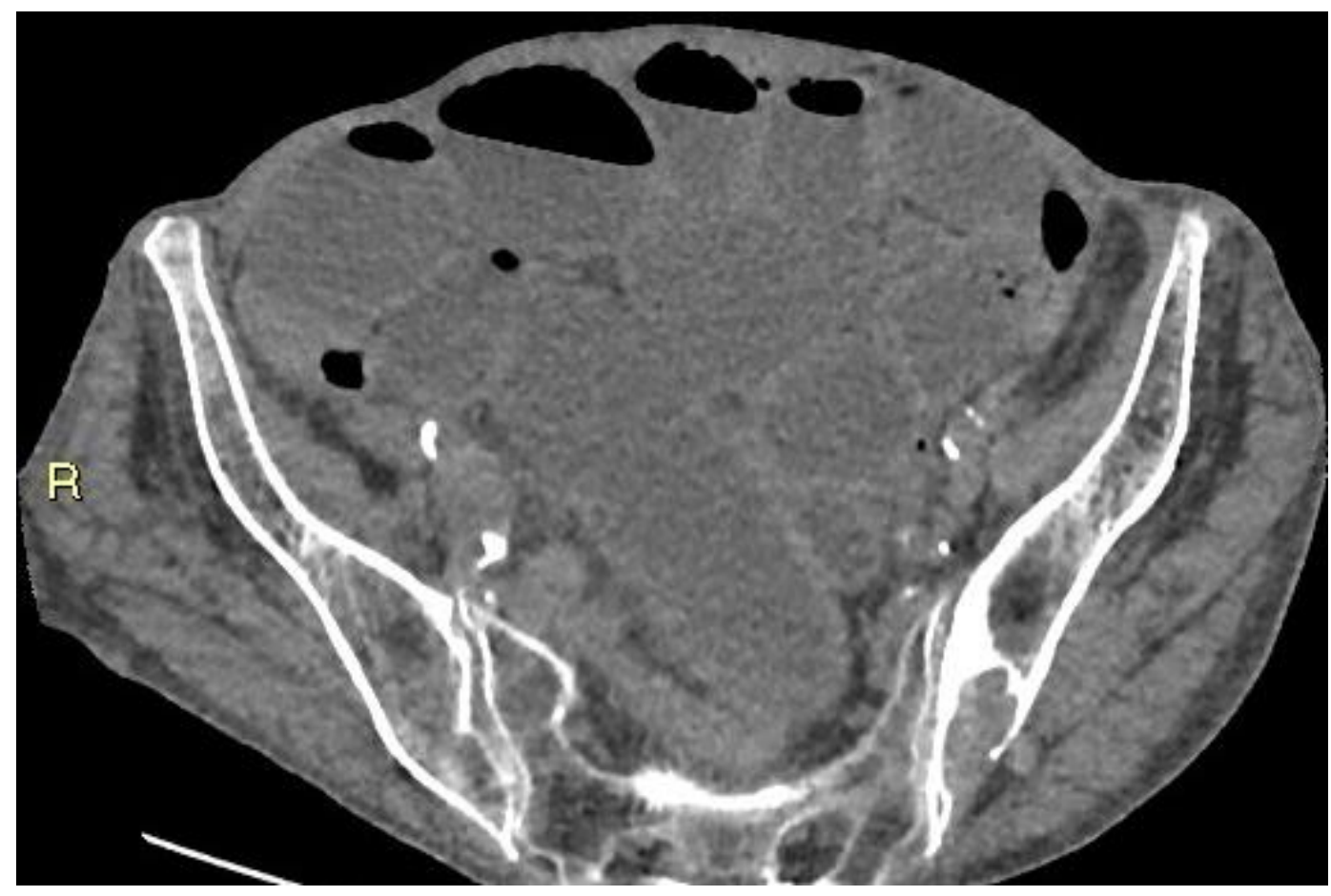

Figure 1. Small bowel luminal distension, with multiple fluid-gas levels, compatible with a small bowel obstruction.

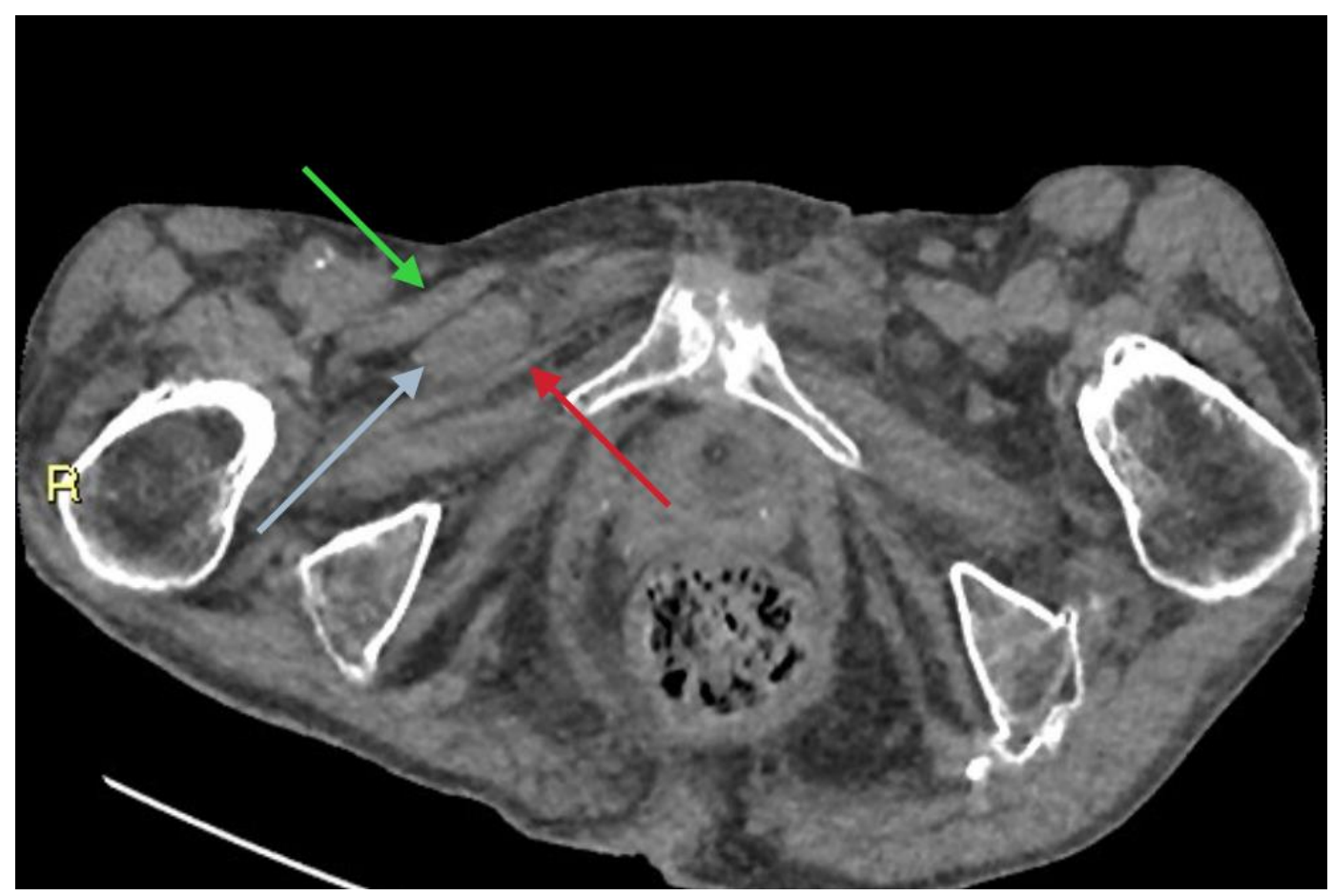

Figure 2. Right obturator hernia (blue arrow), made up of small bowel loop content, lying upon the right pectineus muscle (red arrow), beneath the external obturator muscle (green arrow).

hernia: a case report with literature review. Pan Afr Med J 2019;32:144. 


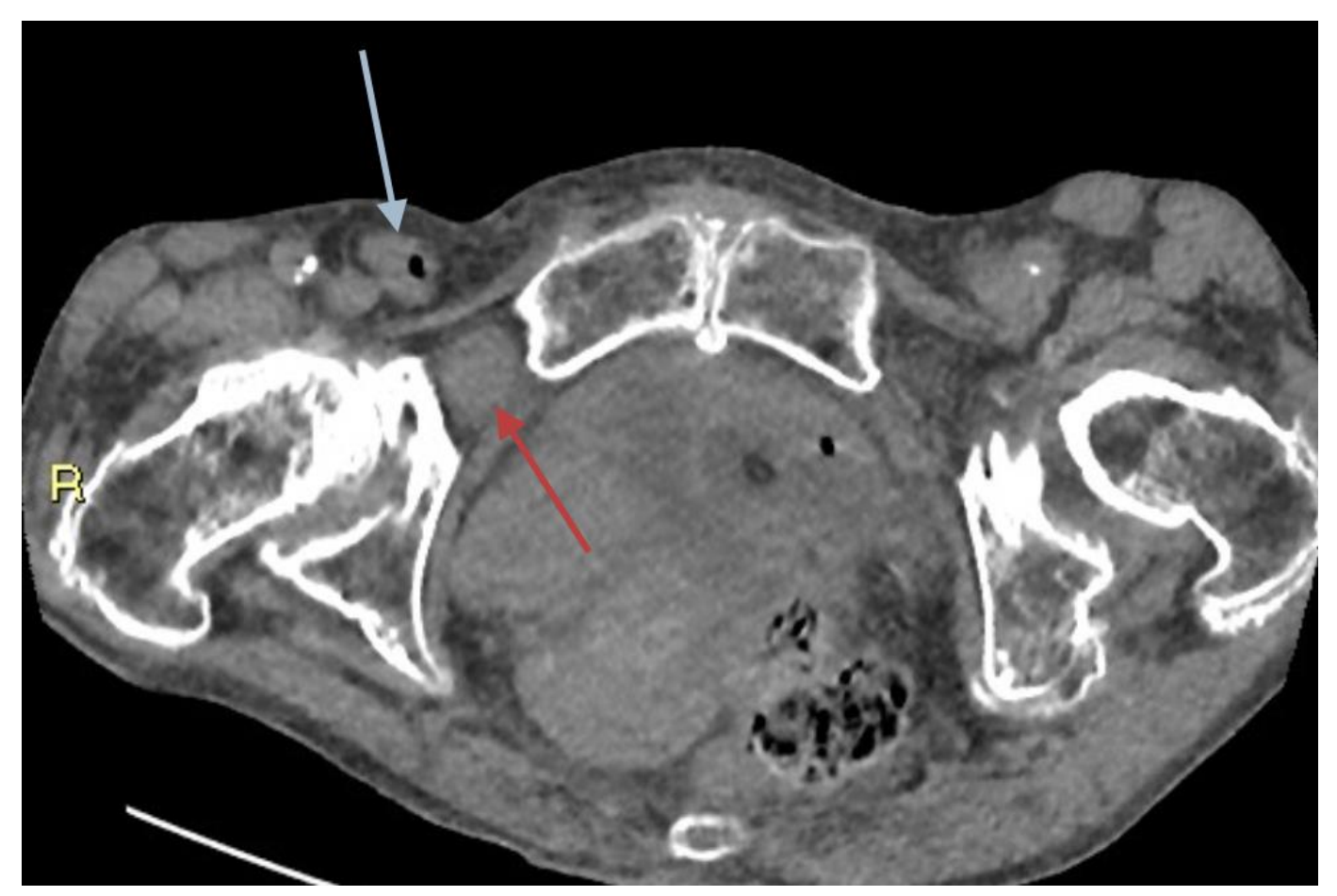

Figure 3. Concurrent ipsilateral right obturator (red arrow) and inguinal (blue arrow) herniæ.

[3] Internal Hernias in the Era of Multidetector CT: Correlation of Imaging and Surgical Findings

Satoshi Doishita, RadioGraphics 2016 36:1, 88-106

[4]. Chitrambal TG, Christopher PJ. Diagnostic difficulties in obturator hernia: a rare case presentation and review of literature. BMJ Case Rep. 2020;13(9):e235644. https://doi.org/10.1136/bcr-2020-235644.

[5]. Droukas DD, Zoland MP, Klein DA. Radiographic and surgical findings of type I obturator hernias in patients with refractory groin pain. Clin Imaging. 2019;55:35-40.

[6]. Li, Z., Gu, C., Wei, M. et al. Diagnosis and treatment of obturator hernia: retrospective analysis of 86 clinical cases at a single institution. BMC Surg 21, 124 (2021). https://doi.org/10.1186/s12893-021-01125-2. 
[7]. Munoz-Forner E, Garcia-Botello S, Lopez-Mozos F, et al. Computed tomographic diagnosis of obturator hernia and its surgical management: a case series. Int J Surg $2007 ; 5: 139-42$.

[8]. Chan KV, Chan CK, Yau KW, et al. Surgical morbidity and mortality in obturator hernia: a 10-year retrospective risk factor evaluation. Hernia 2014;18:387-92.

[9] Chitrambalam TG, Christopher PJ, Sundaraj J, et al. Diagnostic difficulties in obturator hernia: a rare case presentation and review of literature, BMJ Case Reports CP 2020;13:e235644 